\title{
BOUNDS FOR THE DISCRETE PART OF THE SPECTRUM OF A SEMI-BOUNDED SCHRÖDINGER OPERATOR
}

\section{ARNE PERSSON}

\section{Introduction.}

Let $\Omega$ be an unbounded subset of the real $n$-dimensional Cartesian space $R^{n}$. Denote by $L^{2}(\Omega)$ the Hilbert space of square integrable complex-valued functions on $\Omega$ with the scalar product

$$
(u, v)=\int_{\Omega} u(x) \overline{v(x)} d x
$$

and the norm $|u|=(u, u)^{\sharp}$.

Let $a(x)$ be a real measurable function, which is bounded from below for all sufficiently large $|x|$. If $a(x)$ also satisfies a certain local condition (see (1.1)), the differential operator in $L^{2}(\Omega)$

$$
P=-\Delta+a(x): \quad \Delta=\sum_{i=1}^{n} \frac{\partial^{2}}{\partial x_{i}{ }^{2}},
$$

with domain consisting of functions vanishing at the boundary of $\Omega$, is seen to be self-adjoint and bounded from below. Define $d_{P}$ as the largest real number below which the spectrum of $P$ is discrete or empty. If $P$ has discrete spectrum, we put $d_{P}=+\infty$. Denote by $S_{r}$ the intersection of $\Omega$ with the sphere $|x| \leqq r$. We shall prove (theorem 2.1) that

$$
d_{P}=\lim _{r \rightarrow \infty} l_{r},
$$

where $l_{r}$ equals the infimum of $(P f, f)$ when $f$ ranges over all regular functions with $|f|=1$ and with compact supports in $\Omega-S_{r}$. An analogous result holds for more general differential operators and boundary problems.

An immediate corollary of $(1)$ is that, if $a(x) \rightarrow+\infty$ as $|x| \rightarrow \infty$, then $d_{P}=+\infty$, that is, the spectrum of $P$ is discrete. This is the classical criterion of H. Weyl (cf. [11]). We shall also, in section 3, apply our

Received April 26, 1960. 
theorem to some other special cases, where further assumptions about the potential $a(x)$ and the shape of $\Omega$ make it easy to estimate the numbers $l_{r}$, and consequently also $d_{P}$.

Results similar to those appearing in section 3 have been obtained by Glazman [4] [5] and Molčanov [7]. They will be referred to in the text. Birman [2] has treated the case when $a(x)$ is bounded from above.

The problem of section 2 was put to me by professor Lars Gårding, for whose interest and valuable criticism I wish to express my gratitude.

\section{The space $\boldsymbol{H}$.}

If $\omega$ is any domain in $R^{n}$, we shall denote by $\mathscr{D}(\omega)$ the set of infinitely differentiable complex-valued functions with compact supports in $\omega$, and by $L^{2}(\omega)$ the Hilbert space of all (equivalence classes of) square integrable functions in $\omega$. The scalar product and the norm in $L^{2}(\omega)$ are defined by

$$
(u, v)_{\omega}=\int_{\omega} u(x) \overline{v(x)} d x \text { and }|u|_{\omega}=(u, u)_{\omega}^{\frac{1}{2}} .
$$

Here $d x=d x_{1} \ldots d x_{n}$ is the ordinary volume element in $R^{n}$. When $\omega=\Omega$ we omit the indices. Similarly we write $\mathscr{D}$ and $L^{2}$ instead of $\mathscr{D}(\Omega)$ and $L^{2}(\Omega)$. We shall also use the notations

$$
(\nabla f, \nabla g)_{\omega}=\int_{w} \sum_{i=1}^{n} \frac{\partial f}{\partial x_{i}} \frac{\overline{\partial g}}{\partial x_{i}} d x, \quad|\nabla f|_{w}^{2}=(\nabla f, \nabla f)_{w} .
$$

Consider the differential operator

$$
\mathscr{P}=-\Delta+a(x)
$$

with domain $D(\mathscr{P})=\mathscr{D}$. On the real measurable function $a(x)$ we shall impose the following two conditions:

(B) If $0<\varrho<r$, then

$$
a_{0}=\lim _{r \rightarrow \infty}\left(\underset{x \in \Omega-S_{r}}{\operatorname{ess} \inf } a(x)\right)>-\infty .
$$

where $\delta>0$ may be chosen arbitrarily small and $M(\delta)$ depends on $\varrho, r$, and $\delta$ but not on $f$.

Sufficient for $(\mathrm{B})$ is, e.g., that

$$
\int_{K}|a(x)|^{\frac{1}{2} n+\varepsilon} d x<\infty
$$

for every bounded set $K \subset \Omega$ and some $\varepsilon>0$ (see [8], where still more general conditions are given). 
We shall see that $\mathscr{P}$ is bounded from below. In fact, it is readily verified that

$$
(\mathscr{P} f, g)=(\nabla f, \nabla g)+(a f, g), \quad f, g \in \mathscr{D} .
$$

Now take $\varrho$ so large that $a(x)>a_{0}-1$ in $\Omega-S_{\varrho}$. Then, if $r>\varrho$, we get from (1.1)

$$
(\mathscr{P} f, f) \geqq|\nabla f|^{2}+\left(a_{0}-1\right)|f|_{\Omega-S_{\varrho}}{ }^{2}-\delta|\nabla f|_{S_{r}}{ }^{2}-M(\delta)|f|_{S_{r}}{ }^{2},
$$

and hence

$$
(\mathscr{P} f, f) \geqq(1-\delta)|\nabla f|^{2}-\left(\left|a_{0}-1\right|+M(\delta)\right)|f|^{2} .
$$

Choosing $\delta<1$ we obtain the desired result.

Adding a constant to $a(x)$ will not influence our conclusions in the sequel, so we may assume without loss of generality that

$$
(\mathscr{P} f, f) \geqq(f, f)
$$

$$
a_{0}>0 \text {. }
$$

But then it is clear that $((f, g))=(\mathscr{P} f, g)$ becomes a scalar product on $\mathscr{D}$. Denote by $H$ the Hilbert space obtained by completing $\mathscr{D}$ in the corresponding norm $\|f\|=(\mathscr{P} f, f)^{\frac{1}{2}}$. Obviously $H \subset L^{2}$ and it follows from (1.2) that all first order weak derivatives of $u \in H$ are also in $L^{2}$. Thus we may write

$$
\|u\|^{2}=|\nabla u|^{2}+(a u, u)
$$

for all $u \in H$. Moreover, every $u \in H$ vanishes at the boundary of $\Omega$, at least in a generalized sense.

In section 2 we shall make use of the following property of $H$.

LEMMA 1.1. If $\varphi$ is infinitely differentiable and $\varphi$ and $\nabla \varphi$ are both bounded in $\Omega$, then $\varphi u \in H$ for all $u \in H$.

Proof. Clearly it is enough to prove that, if $f_{n} \in \mathscr{D}$ and $\left\|f_{n}-u\right\| \rightarrow 0$ as $n \rightarrow \infty$, then also $\left\|\varphi f_{n}-\varphi u\right\| \rightarrow 0$ as $n \rightarrow \infty$.

By use of (1.1), (1.3), and (1.4) we see that $\left\|f_{n}-u\right\| \rightarrow 0$ implies that the expressions

$$
\left|f_{n}-u\right|, \quad\left|\nabla\left(f_{n}-u\right)\right|, \quad\left(|a|\left(f_{n}-u\right), f_{n}-u\right)
$$

all tend to zero as $n \rightarrow \infty$. But, if $C_{1}$ and $C_{2}$ denote the bounds of $\varphi$ and $\nabla \varphi$, respectively, we have

$$
\begin{aligned}
\left\|\varphi f_{n}-\varphi u\right\|^{2} & =\left|\nabla \varphi\left(f_{n}-u\right)\right|^{2}+\left(a \varphi\left(f_{n}-u\right), \varphi\left(f_{n}-u\right)\right) \\
& \leqq 2 C_{1}{ }^{2}\left|\nabla\left(f_{n}-u\right)\right|^{2}+2 C_{2}{ }^{2}\left|f_{n}-u\right|^{2}+C_{1}{ }^{2}\left(|a|\left(f_{n}-u\right), f_{n}-u\right) .
\end{aligned}
$$

Hence the lemma follows.

Math. Scand. $8-10$ 


\section{The operator $P$.}

It is easy to see that

$$
(u, v)=((R u, v)), \quad u \in L^{2}, \quad v \in H,
$$

defines $R$ as a bounded self-adjoint mapping from the whole of $L^{2}$ onto a dense subset of $H$. Moreover, $P=R^{-1}$ exists and is self-adjoint, $P \supset \mathscr{P}$ and $(P u, u)=((u, u)) \geqq(u, u)$ for every $u$ in the domain $D(P)$ of $P$. The operator $P$ is called the Friedrichs extension of $P$ (see [10, p. 329]).

Let $C(P)$ denote the continuous part of the spectrum of $P$ and put

Define

$$
d_{P}=\min _{\lambda \in C(P)} \lambda .
$$

$$
l_{r}=\inf (P f, f), \quad f \in \mathscr{D}\left(\Omega-S_{r}\right), \quad|f|=1 .
$$

It is clear that $l_{r}$ is a non-decreasing function of $r$, so that $l_{P}=\lim _{r \rightarrow \infty} l_{r}$ exists and $1 \leqq l_{P} \leqq+\infty$.

We shall prove that $d_{P}=l_{P}$. This is carried out in two steps, lemmas 2.1 and 2.2. Lemma 2.1 is very general. It is valid for any semi-bounded self-adjoint operator $P$ in $L^{2}$ with $\mathscr{D} \subset D(P)$. The proof of lemma 2.2 is a modification of a method used by Glazman in [5]. In fact, when $a(x)$ is continuous, this lemma is a consequence of the results in [5].

Lemma 2.1. Let $l_{P}$ and $d_{P}$ be defined as above. Then $l_{P} \geqq d_{P}$.

Proof. We shall use the following fact: if $M$ is a compact subset of $L^{2}\left(R^{n}\right)$, then

$$
\sup _{u \in M} \int_{|x|>r}|u(x)|^{2} d x \rightarrow 0 \text { as } \quad r \rightarrow \infty .
$$

This is easy to prove. For if $\varepsilon>0$ is given, it is possible to find a finite number of functions $\left(f_{v}\right)_{1}^{N}$ in $\mathscr{D}\left(R^{n}\right)$ such that every $u \in M$ satisfies the inequality $\left.\left|u-f_{v}\right|\right|_{n}{ }^{2}<\varepsilon$, for at least one $\nu$. Since all $f_{v}$ vanish outside a fixed sphere about the origin, the assertion follows.

Let $P=\int_{1}^{\infty} \lambda d E_{\lambda}$ be the spectral resolution of $P$. For any $\mu<d_{P}$ put $H_{\mu}=E_{\mu}\left(L^{2}\right)$, and denote by $M_{\mu}$ the closed unit sphere in $H_{\mu}$. By hypothesis, $H_{\mu}$ is of finite dimension and thus $M_{\mu}$ is compact (see [1, p. 29]). Hence, in virtue of (2.1), if $\varepsilon>0$ is given, there exists a number $r$ such that

$$
|u|_{\Omega-S_{r}}<\varepsilon
$$

for every $u \in M_{\mu}$. Now let $f$ be any function in $\mathscr{D}\left(\Omega-S_{r}\right)$ with $|f|=1$. $f \in D(P)$, so that $f=u_{1}+u_{2}$, where $u_{1} \in H_{\mu}$ and $u_{2} \in L^{2} \ominus H_{\mu}$. Since $u_{1}(x)+u_{2}(x)=0$ almost everywhere in $S_{r}$, we also have 


$$
\left|u_{1}(x)\right|^{2}+u_{1}(x) \overline{u_{2}(x)}=0 \quad \text { a.e. in } S_{r} .
$$

Integrating this relation over $S_{r}$ and using the fact that $\left(u_{1}, u_{2}\right)=0$ we get

$$
\left|u_{1}\right|_{S_{r}}^{2}=\left(u_{1}, u_{2}\right)_{\Omega-S_{r}} .
$$

By Schwarz's inequality and (2.2) it then follows that

$$
\left|u_{1}\right|_{S_{r}}^{2} \leqq\left|u_{1}\right|_{\Omega-S_{r}}\left|u_{2}\right|_{\Omega-S_{r}} \leqq \varepsilon\left|u_{2}\right| \leqq \varepsilon .
$$

Thus, if $\varepsilon<1$, we have

and

$$
\left|u_{1}\right|^{2}=\left|u_{1}\right|_{S_{r}}^{2}+\left|u_{1}\right|_{\Omega-S_{r}}{ }^{2} \leqq \varepsilon+\varepsilon^{2}<2 \varepsilon
$$

$$
\left|u_{2}\right|^{2}=|f|^{2}-\left|u_{1}\right|^{2}>1-2 \varepsilon .
$$

Finally, as $P u_{1} \in H_{\mu}$ and $P u_{2} \in L^{2} \ominus H_{\mu}$, it follows that

$$
(P f, f)=\left(P u_{1}, u_{1}\right)+\left(P u_{2}, u_{2}\right) \geqq\left(u_{1}, u_{1}\right)+\mu\left(u_{2}, u_{2}\right) \geqq \mu\left(u_{2}, u_{2}\right)>\mu(1-2 \varepsilon) .
$$

This holds true for every $f \in \mathscr{D}\left(\Omega-S_{r}\right)$ and therefore, because $l_{r}$ increases with $r$,

$$
l_{P} \geqq \mu(1-2 \varepsilon) \text {. }
$$

Since we may take $\mu$ as close to $d_{P}$ as we like and since $\varepsilon>0$ is arbitrary, we conclude that $l_{P} \geqq d_{P}$. The proof is finished.

Lemma 2.2. $l_{P}$ and $d_{P}$ satisfy the inequality $l_{P} \leqq d_{P}$.

Proof. Let $\lambda$ be a given element of $C(P)$. We shall prove that $l_{P} \leqq \lambda$.

Let us first choose a sequence $\left(u_{n}\right)_{1}^{\infty}$ of approximate eigenfunctions in $D(P)$ such that (see $[10$, p. 361])

$$
\begin{aligned}
\left|u_{n}\right| & =1, \quad \text { all } n, \\
u_{n} & \rightarrow 0 \text { weakly as } n \rightarrow \infty, \\
\left|P u_{n}-\lambda u_{n}\right| & \rightarrow 0 \text { as } n \rightarrow \infty .
\end{aligned}
$$

We observe that our choice of $\left(u_{n}\right)_{1}^{\infty}$ implies that

$$
\left|u_{n}\right|_{K} \rightarrow 0 \text { as } n \rightarrow \infty \text {, }
$$

for all bounded $K \subset \Omega$. In order to prove this, keep $K$ fixed and take $S_{r}$ so large that $a(x)>0$ almost everywhere in $\Omega-S_{r}$. Then, in virtue of the properties of $\left(u_{n}\right)_{1}^{\infty}$,

$$
1 \geqq\left|P u_{n}-\lambda u_{n}\right| \geqq\left(P u_{n}, u_{n}\right)-\lambda \geqq\left|\nabla u_{n}\right|^{2}+\left(a u_{n}, u_{n}\right)_{S_{r}}-\lambda
$$

for all sufficiently large $n$. Estimating the term $\left(a u_{n}, u_{n}\right)_{S_{r}}$ by $(1.1)$ with $\delta=\frac{1}{2}$ we get 


$$
\left|\nabla u_{n}\right|^{2} \leqq C
$$

where $C=2\left(1+\lambda+M\left(\frac{1}{2}\right)\right)$. Obviously we make no restriction by assuming that $K$ equals some $S_{e}$. We then have

$$
\left|\nabla u_{n}\right|_{s_{e}}^{2}+\left|u_{n}\right|_{s_{e}}^{2} \leqq C+1 .
$$

Since all $u_{n}$ vanish at the boundary of $S_{e}$ except at the very regular part along $|x|=\varrho$, it follows from a well-known lemma by Rellich ([3, p. 489]) that $\left(u_{n}\right)_{1}^{\infty}$ contains a subsequence $\left(u_{n^{\prime}}\right)_{1}^{\infty}$, which converges in the norm $\left|u_{n^{\prime}}\right|_{S^{\cdot}}$ Because $u_{n^{\prime}} \rightarrow 0$ weakly as $n^{\prime} \rightarrow \infty$, the limit function must be zero, that is, $\left|u_{n^{\prime}}\right|_{S_{e}} \rightarrow 0$ as $n^{\prime} \rightarrow \infty$. But then the original sequence itself must have this property, since otherwise we could get a contradiction by applying the arguments above to a suitable subsequence. Hence (2.3) is proved.

Putting (2.5) into (1.1) we get

$$
\left(|a| u_{n}, u_{n}\right)_{s_{e}} \leqq \delta C+M(\delta)\left|u_{n}\right|_{S_{e}}^{2}, \quad r>\varrho,
$$

and therefore (2.4) is an immediate consequence of (2.3).

Now let $\varrho>0$ be a fixed number and define the infinitely differentiable function $\varphi$ such that

$$
\begin{aligned}
& \varphi(x)=1 \text { in } \Omega-S_{e}, \\
& 0 \leqq \varphi(x) \leqq 1, \\
& \nabla \varphi(x) \text { bounded in } \Omega .
\end{aligned}
$$

Then, by lemma 1.1, $\varphi u_{n} \in H$. We are going to prove that

$$
\left\|\varphi u_{n}\right\|^{2} \leqq \lambda\left|\varphi u_{n}\right|^{2}+o(1),
$$

where $o(1) \rightarrow 0$ as $n \rightarrow \infty$. In order to do this we observe that

$$
\left\|\varphi u_{n}\right\|^{2}=\left|\nabla \varphi u_{n}\right|^{2}+\left(a \varphi u_{n}, \varphi u_{n}\right),
$$

and that the properties of $\left(u_{n}\right)_{1}^{\infty}$ give the relation

$$
\left\|u_{n}\right\|^{2}=\left|\nabla u_{n}\right|^{2}+\left(a u_{n}, u_{n}\right)=\lambda\left|u_{n}\right|^{2}+o(1) .
$$

Moreover, using (2.3)-(2.5) and the assumption about $\varphi$ we obtain for the first term in (2.7)

$$
\left|\nabla \varphi u_{n}\right|^{2}=\left|\varphi \nabla u_{n}\right|^{2}+2 \operatorname{Re}\left(u_{n} \nabla \varphi, \varphi \nabla u_{n}\right)+\left|u_{n} \nabla \varphi\right|^{2} \leqq\left|\nabla u_{n}\right|^{2}+o(1),
$$

and for the second

$$
\left(a \varphi u_{n}, \varphi u_{n}\right)=\left(a u_{n}, u_{n}\right)+o(1) .
$$

Hence, using first (2.8) and thereafter (2.3),

$$
\left\|\varphi u_{n}\right\|^{2} \leqq\left|\nabla u_{n}\right|^{2}+\left(a u_{n}, u_{n}\right)+o(1)=\lambda\left|u_{n}\right|^{2}+o(1)=\lambda\left|\varphi u_{n}\right|^{2}+o(1),
$$

which is exactly (2.6). 
Now let $\varepsilon>0$ be a given number. It follows from the definition of $l_{P}$ that there exists an $S_{r}$ such that

$$
\|f\|^{2} \geqq\left(l_{P}-\varepsilon\right)|f|^{2}
$$

for all $f \in \mathscr{D}\left(\Omega-S_{r}\right)$. By an approximation with functions in $\mathscr{D}\left(\Omega-S_{r}\right)$ (2.9) is seen to hold also when $f \in H$ and the support of $f$ is contained in $\Omega-S_{r}$. Therefore, if we choose $\varphi(x)=0$ in $S_{r}$ and take $\varrho>r,(2.6)$ and (2.9) give the inequality

$$
\left(l_{P}-\varepsilon\right)\left|\varphi u_{n}\right|^{2} \leqq\left\|\varphi u_{n}\right\|^{2} \leqq \lambda\left|\varphi u_{n}\right|^{2}+o(1) .
$$

This together with the fact that $\left|\varphi u_{n}\right| \rightarrow 1$ as $n \rightarrow \infty$ shows that

$$
l_{P} \leqq \lambda+\varepsilon .
$$

Since $\varepsilon>0$ is arbitrary, the lemma follows.

Combining the last two lemmas we immediately get the following theorem.

Theorem 2.1. Let a(x) satisfy (A) and (B) and let $P$ be the Friedrichs extension of $-\Delta+a(x)$ with domain $\mathscr{D}$. Then

$$
d_{P}=l_{P}=\lim _{r \rightarrow \infty}\left(\inf _{\substack{|f|=1 \\ f \in \mathscr{D}\left(\Omega-S_{r}\right)}}(P f, f)\right),
$$

where $d_{P}=\min _{\lambda \in C(P)} \lambda$.

Remark 1. We have assumed in the proofs that $l_{P}<+\infty$. But it is easy to modify them to treat also the case $l_{P}=+\infty$.

REMARK 2. So far we have excluded the case, where $\Omega$ is bounded. However, it is readily verified that in this case $d_{P}=+\infty$. In fact, the set

$$
M=\{u:(P u, u) \leqq C, u \in D(P)\}
$$

is easily seen to be precompact (use (B) with $S_{e}=S_{r}=\Omega$ and Rellich's lemma). Hence the assertion follows from a well-known criterion by Rellich ([9]).

REMaRK 3. It is possible to prove theorem 2.1 for more general operators. For instance, we may start with a hermitean differential form

$$
B f \bar{g}=\sum_{\substack{|\alpha| \leqq m \\|\beta| \leqq m}} a_{\alpha \beta}(x) D_{\alpha} f(x) D_{\beta} \overline{g(x)}, \quad D_{\alpha}=\frac{\partial^{|\alpha|}}{\partial x^{\alpha}}=\frac{\partial^{\alpha_{1}+\ldots+\alpha_{n}}}{\partial x_{1}^{\alpha_{1}} \ldots \partial x_{n}{ }^{\alpha_{n}}},
$$

of double order $m ; m$. The principal part $p$ of $B$ is supposed to be uniformly positive definite and bounded, i.e., 


$$
\varepsilon_{0} \sum_{|\alpha|=m}\left|D_{\alpha} f(x)\right|^{2} \leqq p f \bar{f} \leqq C_{0} \sum_{|\alpha|=m}\left|D_{\alpha} f(x)\right|^{2} .
$$

If we put $a=B-p$, and $a$ has properties corresponding to (A) and (B), that is,

and

$$
\lim _{r \rightarrow \infty}\left(\inf _{|f| \Omega-S_{r}=1} \int_{\Omega-S_{r}} a f \bar{f} d x\right)>-\infty
$$

$$
\left|\int_{S_{r}} a f \bar{f} d x\right| \leqq \delta \int_{S_{r}} \sum_{|\alpha|=m}\left|D_{\alpha} f(x)\right|^{2} d x+M(\delta)|f|_{S_{r}}{ }^{2},
$$

then $B$ is bounded from below. After addition of a suitable constant it follows that

$$
(B f, f)=\int B f \bar{f} d x \geqq(f, f),
$$

and hence, if we complete $\mathscr{D}$ in the norm $((f, f))^{\frac{1}{2}}=(B f, f)^{\frac{1}{2}}$, we get a Hilbert space $H \subset L^{2}$. It is easily verified that the relation

$$
(P u, v)=((u, v))
$$

defines $P$ as a semi-bounded self-adjoint operator in $L^{2}$. All results in this section may be applied to this operator. The proofs will contain nothing essentially new, so we do not go into details.

RemarK 4. Even more general boundary problems may be treated in the same manner, as soon as (B) is fulfilled for every $f \in \mathscr{E}$, where $\mathscr{E}$ is the set of smooth functions defining the boundary problem in question. We only have to change the definition of $l_{P}$ by taking infimum over those functions in $\mathscr{E}$ which have their supports in $\Omega-S_{r}$.

Remark 5. Let $P$ be a formally self-adjoint hypoelliptic operator with constant coefficients defined in any domain $\Omega$ in $R^{n}$. Then $P$ is semi-bounded (see [6, p. 233]). Let $P$ be any semi-bounded self-adjoint extension. Using the methods of this paper it is possible to prove that

$$
d_{P}=\lim _{K \rightarrow \Omega}\left(\inf _{\substack{|f|=1 \\ f \in D(P)}}(P f, f)\right), \quad \text { the support of } f \text { contained in } \Omega-K,
$$

where $K$ runs through an increasing sequence of compact subsets of $\Omega$. However, in proving the important relation (2.3) one has to use a Fourier transform and Arzelà's theorem (cf. [6, p. 202]).

\section{Applications.}

It is immediately seen from theorem 2.1 that

$$
d_{P} \geqq a_{0}=\lim _{r \rightarrow \infty}\left(\underset{x \in \Omega-S_{r}}{\operatorname{essinf}} a(x)\right) .
$$


However, special assumptions about $a(x)$ and the shape of $\Omega$ make it possible to get more precise information. In particular we are able to generalize (proposition 3.1 and 3.2) the following result, appearing in [4] (see also [5]): if $\Omega=R^{n}, a(x)$ is continuous, and $a_{0}=\lim _{|x| \rightarrow \infty} a(x)$ exists, then $d_{P}=a_{0}$. Proposition 3.3 gives a sufficient condition on $\Omega$ for the spectrum of $P$ to be discrete. More precise results in this direction are proved in [6]. Before stating the results we shall give two definitions and make some preliminary remarks.

For any open set $\Omega \subset R^{n}$, put $b_{\Omega}=\sup \varrho_{\Sigma}$, where $\varrho_{\Sigma}$ denotes the radius of the sphere $\Sigma$ and $\Sigma$ runs through all spheres contained in $\Omega$. We allow $b_{\Omega}$ to be $+\infty$.

Definition 3.1. $\Omega$ is said to be regular of order $\varkappa(1 \leqq \varkappa<\infty)$, if every compact set $K \subset \Omega$ can be covered by a finite number of closed parallelepipeds $\left(E_{v}\right)_{1}^{N}$ in such a way that

1) every $E_{v}$ has at least one side that does not meet $K$,

2) the altitude of $E_{\nu}$ against this side is less than or equal to $b_{\Omega}$,

3) every $x \in K$ belongs to at most $\varkappa$ of the $E_{v}$ 's.

LeMma 3.1. If $\Omega$ is regular of order $\varkappa$, then

$$
|f|^{2} \leqq \varkappa \cdot \frac{1}{2} b_{\Omega}{ }^{2}|\nabla f|^{2}, \quad f \in \mathscr{D} .
$$

Proof. Let $f \in \mathscr{D}$. Since $f$ has compact support, it is clearly enough to show that

$$
|f|_{E}{ }^{2} \leqq \frac{1}{2} b_{\Omega}{ }^{2}|\nabla f|_{E}{ }^{2}
$$

for any parallelepiped with the properties 1) and 2). But the inequality (3.3) is well known and easily proved. In fact, choose a coordinate system $\left(y_{1}, \ldots, y_{n}\right)$ such that $E$ is contained in $y_{1} \geqq 0$ and such that the side that does not meet the support of $f$ is contained in $y_{1}=0$. Applying Schwarz's inequality to the identity

we obtain

$$
f(y)=\int_{0}^{y_{1}} f_{y_{1}}\left(t, y_{2}, \ldots, y_{n}\right) d t
$$

$$
|f(y)|^{2} \leqq y_{1} \int_{0}^{y_{1}}\left|f_{y_{1}}\right|^{2} d t \leqq y_{1} \int_{0}^{b}|\nabla f|^{2} d y_{1},
$$

$b$ being the altitude of $E$. If we now integrate over $E,(3.3)$ follows. Hence the lemma is proved.

When $\Omega$ is a sphere with radius $\varrho$, we shall denote by $\lambda_{e}$ the best possible constant appearing in (3.2). The number $\lambda_{e}$ is the smallest 
eigenvalue of the operator $-\Delta$ with vanishing boundary values in $\Omega$. Moreover, $\lambda_{\ell} \rightarrow \infty$ as $\varrho \rightarrow \infty$ and $\lambda_{e}$ is a continuous function of $\varrho$.

We borrow the following definition from Glazman [4].

Definition 3.2. $\Omega$ is said to be quasi-conical, if it contains arbitrarily large spheres. $\Omega$ is called quasi-cylindrical, if it is not quasi-conical, but it contains an infinite sequence of disjoint spheres with fixed positive radius. In this case we also define the radius of $\Omega$ as being the supremum of all such radii. Finally, we say that $\Omega$ is quasi-bounded, if it is neither quasi-conical nor quasi-cylindrical.

Thus every domain $\Omega$ belongs to exactly one of these three classes. It is clear that a quasi-conical domain is regular of order 1 .

Proposition 3.1. Let $\Omega$ be quasi-conical and let $a(x)$ have the limit $a_{0}$ on a quasi-conical part $\omega$ of $\Omega$. Then $d_{P}=a_{0}$.

Proof. In virtue of (3.1), it is sufficient to prove that $l_{P} \leqq a_{0}$. By assumption, for any given $r$, the set $\omega-S_{r} \cap \omega$ contains a sphere $\Sigma$ with arbitrarily large radius $\varrho$ such that $a(x)<a_{0}+\varepsilon$ in $\Sigma$. Moreover, we can choose $g \in \mathscr{D}(\Sigma)$ such that $|g|=1$ and

It follows that

$$
|\nabla g|^{2} \leqq \lambda_{e}^{-1}+\varepsilon .
$$

$$
l_{r} \leqq|\nabla g|^{2}+(a g, g) \leqq \lambda_{\varrho}^{-1}+a_{0}+2 \varepsilon
$$

for all $r$, and consequently

$$
l_{P} \leqq \lambda_{Q}^{-1}+a_{0}+2 \varepsilon .
$$

Letting $\varrho \rightarrow \infty$ and $\varepsilon \rightarrow 0$ we get the desired result.

Proposition 3.2. Let $\Omega$ be a quasi-cylindrical domain, which has radius $\alpha$ and is regular of order $\varkappa$. If $a_{0}=\lim _{|x| \rightarrow \infty} a(x)$ exists, then $d_{P}=a_{0}+\mu$, where $2 \varkappa^{-1} \alpha^{-2} \leqq \mu \leqq \lambda_{\alpha}{ }^{-1}$.

Proof. Given $\varepsilon>0$, we can find $R$ such that $\left|a(x)-a_{0}\right|<\varepsilon$ in $\Omega-S_{r}$ and $b_{\Omega-S_{r}} \leqq \alpha+\varepsilon$ whenever $r>R$. Hence, on account of lemma 3.1,

$$
l_{r} \geqq 2 \varkappa^{-1}(\alpha+\varepsilon)^{-2}+a_{0}-\varepsilon, \quad \text { all } r>R .
$$

On the other hand, let $\left(\Sigma_{v}\right)_{1}^{\infty}$ be a sequence of spheres in $\Omega$ with common radius larger than $\alpha-\varepsilon$. Take out one $\Sigma_{\nu}$ situated in $\Omega-S_{r}$. Then, by a convenient choice of $g \in \mathscr{D}\left(\Sigma_{p}\right)$, we get

$$
l_{r} \leqq\left(\lambda_{\alpha}-\varepsilon\right)^{-1}+\varepsilon+a_{0}+\varepsilon, \quad \text { all } r>R .
$$

Obviously, these two inequalities together imply the proposition. 
Proposition 3.3. If $\Omega$ is quasi-bounded and regular of order $x<+\infty$, then the spectrum of $P$ is discrete.

Proof. We shall prove that $\lim _{r \rightarrow \infty} l_{r}=+\infty$. Given $\varepsilon>0$, there exists $R$ such that $a(x) \geqq 0$ in $\Omega-S_{r}$ and $b_{\Omega-S_{r}} \leqq \varepsilon$ for all $r>R$. Hence

$$
l_{r} \geqq 2 \varkappa^{-1} \varepsilon^{-2}, \quad r>R,
$$

which proves our assertion.

Remark. A necessary and sufficient condition for discrete spectrum when $a(x)$ is continuous and $\Omega$ is arbitrary was given by Molčanov [7].

\section{BIBLIOGRAPHY}

1. N. Bourbaki, Espaces vectoriels topologiques, Ch. I-II (Act. Ind. Sci. 1189), Paris, 1953.

2. M. Sh. Birman, On the spectrum of Schrödinger's and Dirac's operators, Dokl. Akad. Nauk SSSR 129 (1959), 239-245. (Russian.)

3. R. Courant und D. Hilbert, Methoden der mathematischen Physik II, Berlin, 1937.

4. I. M. Glazman, On the character of the spectrum of many-dimensional singular boundary problems, Dokl. Akad. Nauk SSSR 87 (1952), 171-174. (Russian.)

5. I. M. Glazman, On the application of the method of decomposition to many-dimensional singular boundary problems, Mat. Sb. N.S. 35 (77) (1954), 231-246. (Russian.)

6. L. Hörmander, On the theory of gereral partial differential operators, Acta Math. 94 (1955), 161-248.

7. A. M. Molčanov, Criterion for discrete spectrum of semi-bounded Sturm-Liouville operators, Trudy Moskov. Mat. Obšč. 2 (1953), 169-199. (Russian.)

8. N. Nilsson, Essential self-adjointness and the spectral resolution of Hamiltonian operators, Kungl. Fysiogr. Sällsk. i Lund Förh. 29 (1959), 1-19.

9. F. Rellich, Störungstheorie der Spektralzerlegung, Math. Ann. 118 (1942), 462-484.

10. F. Riesz et B. Sz.-Nagy, Leçons d'Analyse fonctionnelle, Budapest, 1952.

11. H. Weyl, Uber gewöhnliche Differentialgleichungen mit Singularitäten und die zugehörigen Entwicklungen willkürlicher Funktionen, Math. Ann. 68 (1910), 220-269. 